\title{
Effect of mangosteen (Garcinia mangostana L.) pericarp extract on the different carriers for antioxidant active packaging films
}

\begin{abstract}
Active packaging with the addition of natural antioxidant compounds has been developed as a potential alternative to plastic-based materials to protect food products from environmental damages. This study investigated the effect of mangosteen (Garcinia mangostana L.) pericarp extract (MPE) as a natural antioxidant on the physicochemical properties of fish gelatin (FG), soy protein isolates (SPI), and corn starch (CS) films. MPE at different concentrations $(0,1,3$, and $5 \%, \mathrm{w} / \mathrm{v}$ ) were added into each of the film-forming solutions (FFS) and film without the active compound was considered as a control. MPE increased the water vapor permeability and reduced the mechanical properties of the films. In addition, protein-based films resulted in better mechanical properties compared to starch-based films. However, corn starch films possessed good water resistance than protein-based films. SPI film with 5\% MPE expressed the highest value of DPPH-scavenging activity (92.67\%) followed by CS (88.43\%) and FG (61.51\%) films. In conclusion, MPE added in the FFS has improved the functional properties of biodegradable films with soy protein-based films exhibited the highest antioxidant capacity and, therefore, could be a promising natural antioxidant to be incorporated in packaging material as active film.
\end{abstract}

Keyword: Active packaging; Fish gelatin; Soy protein isolate; Corn starch; Mangosteen pericarp extract 